\title{
Astrometry of the Planetary-System Millisecond Pulsar B1257+12
}

\author{
N. V. Nunes \& N. Bartel \\ York University, North York, Canada
}

\begin{abstract}
We present the VLBI determined position of the millisecond pulsar PSR B1257+12 which has been shown to have a planetary system (Wolszczan \& Frail 1992). The position determination for epoch June 20, 1992 is: $\alpha_{J 2000}=13^{\mathrm{h}} 0^{\mathrm{m}} 3^{\mathrm{s}} 05005(5), \delta_{J 2000}=+$ $12^{\circ} 40^{\prime} 56^{\prime \prime} .7043(34)$. We indicate how the combination of such pulsar observations along with timing observations can be used to directly tie the solar-system dynamic reference frames and the extragalactic reference frame.
\end{abstract}

\section{Observations and Data Analysis}

The millisecond pulsar PSR B1257+12 and the two extragalactic reference sources $1257+145$ and $1252+119$, shown in Fig. 1, were observed in left circular polarization at $18 \mathrm{~cm}(1.667 \mathrm{GHz})$ on 1992 June $20 / 21$. We used the following radio telescopes: $100 \mathrm{~m}$ Effelsberg radio antenna, $70 \mathrm{~m}$ Goldstone DSN antenna, $70 \mathrm{~m}$ Madrid DSN antenna and the Very Large Array. The data were recorded using the Mark IIIA VLBI system in mode A obtaining a bandwidth of $\sim 56 \mathrm{MHz}$. Interleaved observations were made with each cycle consisting of a $10 \mathrm{~min}$ scan on the pulsar, $0.5 \mathrm{~min}$ scan on each reference source and 1-2 min antenna slewing time between the scans. The observations were correlated at the Haystack Observatory where gating of the processor was used to increase the signal-to-noise ratio of the pulsar. The data were then analyzed using the astrometry package ASPY (cf. Ebbers \& Bartel 1995) and the technique described by Bartel et al. (1986). The post-fit phase delay residuals from our best solution have a weighted rms of $0.1 \mathrm{~ns}$ and are shown for one baseline in Fig. 2. The position determination for PSR B $1257+12$ corresponding to this solution is:

$$
\alpha_{J_{2000}}=13^{\mathrm{h}} 0^{\mathrm{m}} 3^{\mathrm{s}} .05005(5), \quad \delta_{J 2000}=+12^{\circ} 40^{\prime} 56^{\prime \prime} .7043(34) .
$$

where the uncertainties are one standard error and have been estimated using the method described in Bartel et al. (1996).

\section{Comparison with Other Position Determinations}

Timing positions are available for PSR B1257+12 w.r.t. PEP740R (Wolszczan \& Frail 1992; Wolszczan 1994a, 1994b) and DE200 (Wolszczan pers. comm. 1996). Before these can be compared to our VLBI position, all coordinates must be rotated into the same reference frame. This was done using the frame conversion of Bartel et al. (1996), which includes the indirect frame tie of Folkner et al. (1994). All positions are plotted in Fig. 3 after being propagated to our observation epoch. Timing positions $1 \& 2$ are within 2 times their uncertainties of our VLBI position, while timing positions $3 \& 4$ are in good agreement with ours, given the 3 mas uncertainty in the frame tie. In fact, the uncertainties in these are sufficiently small as to allow a more accurate and direct frame tie to be determined. This requires an accurate position determination of at least one more suitable millisecond pulsar. Using the position determinations of PSR B1937+21 (see Bartel et al. 1996), along with a small improvement in accuracy, will allow a direct frame tie between the extragalactic and the solarsystem dynamic reference frames to be computed with an accuracy of 1-2 mas. 


\section{References}

Bartel, N., et al. 1986. Nature, 319, 733-738.

Bartel, N., et al. 1996. $A J, 112,1690-1696$.

Ebbers, A., \& Bartel, N. 1995. JRASC, 89/4, 186.

Folkner, W. M., et al. 1994. A\&A, 287, 279-289.

Wolszczan, A., \& Frail, D. A. 1992. Nature, 355, 145-147.

Wolszczan, A. 1994a. ApESS, 212, 67-75.

Wolszczan, A. 1994b. Science, 264, 538-542.

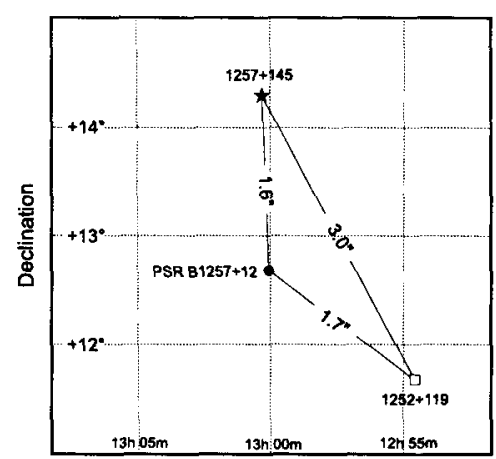

Right Ascension

Figure 1. Sources observed, with their J2000.0 positions and separations on the sky.

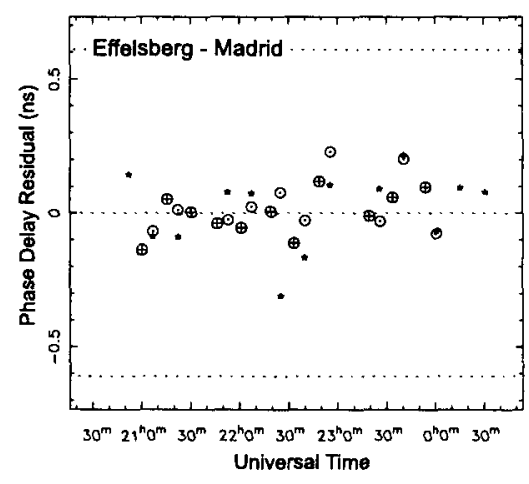

Figure 2. Residuals for EffelsbergMadrid baseline. Symbols are: $\star 1257+145$, $\odot$ difference PSR $1257+145$ and $\oplus$ difference $1252+119$ PSR.

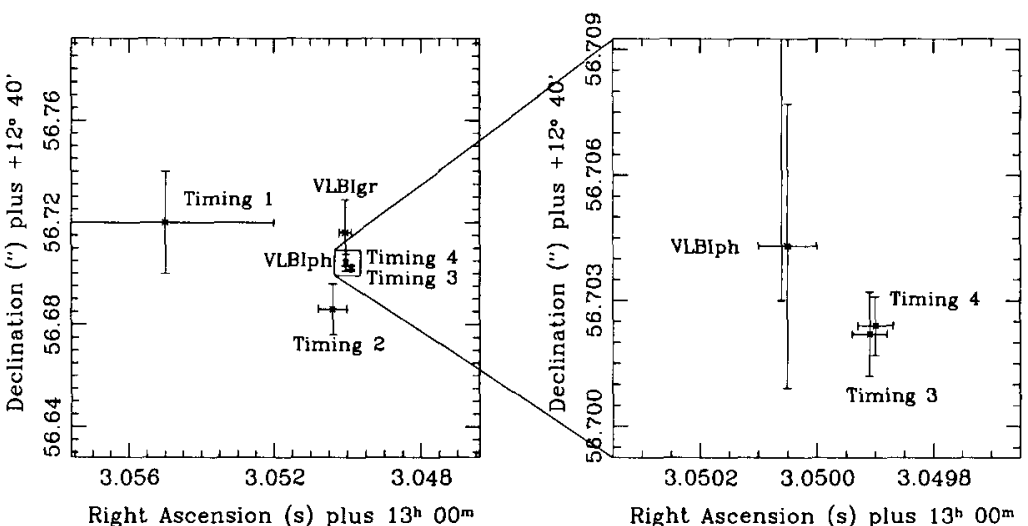

Figure 3. Comparison of PSR B1257+12 position determinations. Timing positions are from Wolszczan \& Frail 1992 (1), Wolszczan 1994a (2), Wolszczan 1994b (3) and Wolszczan pers. comm. 1996 (4). VLBIph is our solution using phase delays while, for comparison, VLBIgr is the solution using group delays. 